\title{
OBESIDADE E SINTOMAS DE DEPRESSÃO, ANSIEDADE E DESESPERANÇA EM MULHERES SEDENTÁRIAS E NÃO SEDENTÁRIAS
}

\author{
OBESITY AND SYMPTOMS OF DEPRESSION, ANXIETY AND HOPELESSNESS \\ IN SEDENTARY AND NON-SEDENTARY WOMEN.
}

Ana P. Tosetto ${ }^{1}$, Carlos A. Simeão Júnior ${ }^{2}$

\begin{abstract}
${ }^{1}$ Docente, Departamento de Ciências Biológicas e da Saúde, Centro Universitário Moura Lacerda - Ribeirão Preto. Professora de Educação Física, Prefeitura Municipal da Estância Turística de Batatais. Mestre em Ciências (Psicobiologia), Departamento de Psicologia e Educação, Faculdade de Filosofia, Ciências e Letras Ribeirão Preto - USP. Psicóloga Clínica. ${ }^{2}$ Docente. Departamento de Ciências Biológicas e da Saúde, Centro Universitário Moura Lacerda - Ribeirão Preto. Docente, Instituto de Ciências da Saúde da Universidade Paulista. Mestre e Doutorando em Alimentos e Nutrição, Departamento de Alimentos e Nutrição - FCFAR. Universidade Estadual Paulista/ Araraquara.
\end{abstract}

Correspondência: Ana Paula Tosetto. Rua Tibiriçá, 682, apto. 61, 14010 - 090, Ribeirão Preto, São Paulo, Brasil. (E-mail: tosetto @ usp.br)

Tosetto AP, Simeão Júnior CA. Obesidade e sintomas de depressão, ansiedade e desesperança em mulheres sedentárias e não sedentárias. Medicina (Ribeirão Preto) 2008; 41 (4): 497-507.

RESUMO: O objetivo deste estudo de campo foi verificar se existe relação entre obesidade e sintomas de depressão, ansiedade e desesperança em 40 mulheres, com idade média $=30,35$ anos $( \pm 8,60)$, divididas em dois grupos: não sedentárias, caracterizadas por praticar atividade física pelo menos três vezes semanais e por três semanas consecutivas e sedentárias, caracterizadas por não praticar qualquer tipo de atividade física regular quando recrutadas. O método consistiu em: avaliações objetivas do humor, através dos Inventários Beck de Ansiedade (BAI), de Depressão (BDI) e de Desesperança (BHS) e Avaliação Física, incluindo massa corporal total, estatura, circunferências da cintura e do quadril e espessura de dobras cutâneas. Foram realizados cálculos do Índice de Massa Corpórea (IMC), da Razão Cintura-Quadril (RCQ) e da porcentagem de gordura corporal (\%G) para avaliar presença e grau de obesidade. Resultados das análises de regressão para mínimos quadrados sustentaram as hipóteses iniciais quanto a existência da relação entre obesidade e sintomas psíquicos somente em mulheres sedentárias $(B D I / R C Q, p=0,035, B D I / I M C, p=0,009, B D I / \% G, p=0,019, B A I / I M C, p=0,009, B A I / \% G, p=0,037$, $\mathrm{BHS} / \mathrm{RCQ}, \mathrm{p}=0,025, \mathrm{BHS} / \mathrm{IMC}, \mathrm{p}=0,041)$, já que a relação de dependência não pôde ser confirmada em mulheres não sedentárias (BDI/RCQ, $p=0,750, B D I / I M C, p=0,141, B D I / \% G, p=0,064, B A I /$ $R C Q, p=0,729, B A I / I M C, p=0,384, B A I / \% G, p=0,246, B H S / R C Q, p=0,491, B H S / I M C, p=0,986, B H S /$ $\% \mathrm{G}, \mathrm{p}=0,322)$ e que, quanto maior o nível de obesidade, maiores os níveis de sintomas psíquicos nos dois grupos. Essas observações parecem indicar que a prática de atividade física foi um fator de minimização da presença e intensidade de sintomas psíquicos em mulheres não sedentárias.

Descritores: Obesidade. Sintomas Psíquicos. Atividade Física. 


\section{1- INTRODUÇÃO}

Nos tempos atuais, frequientemente se tem discutido temas relacionados aos distúrbios alimentares, bem como sua necessidade de diagnósticos e tratamentos adequados, enfatizando a importância da abordagem multiprofissional e do trabalho interdisciplinar na prevenção e no tratamento. Dentre os distúrbios alimentares, a obesidade tornou-se um problema de saúde pública mundial, considerada uma doença epidemiológica, que atinge qualquer ser humano, independente do gênero, condição intelectual, nível socioeconômico e idade ${ }^{1}$.

Tanto os países desenvolvidos como os países em desenvolvimento apresentam aumento de sua incidência. Atualmente, o fenômeno da transição nutricional, na qual a obesidade desponta como um problema mais frequiente e mais grave que a desnutrição, sobrecarrega nosso sistema de saúde com uma demanda crescente por atendimento a diversas doenças crônicas relacionadas com a obesidade, como doenças cardiovasculares, diabetes mellitus e hipertensão ${ }^{2,3}$.

A ampliação das facilidades decorrentes da tecnologia moderna, o sedentarismo e as mudanças significativas no estilo de vida tornaram-se causas para o desenvolvimento de doenças ou riscos à saúde humana, especialmente o favorecimento e a manutenção de alguns tipos de obesidade. Esses fatores, associados à falta de adequação alimentar e ao estresse, incluem-se entre os principais determinantes da obesidade ${ }^{4,5,6}$.

Portanto, processos bio-psicossociais e históricos devem ser considerados para sua ocorrência e, sobretudo, o estilo de vida, que inclui não só os hábitos alimentares, como a prática de atividades físicas, controle emocional estável e cognições funcionais ${ }^{7,1}$.

Além dos prejuízos para a saúde física, a presença de sintomas depressivos, ansiedade, distúrbios alimentares e transtornos de personalidade pode ser observada em pacientes obesos ${ }^{8}$. Tal observação parece ter uma relação diretamente proporcional ao índice de massa corporal (IMC) ${ }^{9}$.

Um estudo envolvendo 50 pacientes com obesidade grave (IMC igual ou superior a 40) apontou uma alta incidência do transtorno da compulsão alimentar periódica e de episódios isolados de compulsão alimentar, bem como de sintomas de ansiedade, depressão e de grau elevado de preocupação com a imagem corporal ${ }^{10}$.
Evidências científicas consistentes indicam que a atividade física regular traz amplos benefícios para a saúde física e mental ${ }^{11}$, aumentando sua participação na promoção da saúde e na prevenção de doenças $^{12}$. Estudos epidemiológicos e experimentais demonstram uma correlação positiva entre a atividade física e a diminuição da mortalidade, sugerindo também um efeito positivo não só nos riscos de enfermidades cardiovasculares e no perfil dos lipídeos plasmáticos, como também na manutenção da densidade óssea, na redução das dores lombares, melhores perspectivas para o controle de enfermidades respiratórias crônicas e diabetes, tratamento primário da arteriosclerose e menor incidência de certos tipos de câncer em indivíduos ativos ${ }^{3}$.

Quanto aos benefícios para a saúde mental em decorrência da atividade física regular, tem sido observado melhora de humor e auto-conceito a curto prazo e diminuição da ansiedade, do estresse e da depressão a longo prazo ${ }^{3}$. Além disso, a associação da psicoterapia cognitiva ao tratamento da obesidade permite resultados mais efetivos quanto às mudanças no repertório de comportamentos relacionados aos hábitos alimentares, padrões errôneos de raciocínio, além de atuar sobre o contexto, tornando-o facilitador da perda e manutenção do peso ${ }^{13-16}$.

Estudo recente utilizando um programa de terapia cognitivo-comportamental em grupo com 21 pacientes obesos com transtorno da compulsão alimentar periódica apontou melhora significativa da compulsão alimentar, do peso corporal, da preocupação com a forma corporal e dos sintomas depressivos associados ao transtorno, avaliada no início do tratamento e no final do estudo ${ }^{17}$. Porém, sua eficácia na redução do peso corporal e a manutenção dos efeitos a longo prazo ainda precisam ser melhor investigadas ${ }^{18}$.

Portanto, o tratamento da obesidade continua produzindo resultados insatisfatórios, em grande parte por estratégias equivocadas e pelo mau uso dos recursos terapêuticos disponíveis. Além da busca por tratamentos mais eficazes, é necessário que sejam adotadas medidas de prevenção para conter o surgimento de casos novos e evitar que a incidência da doença continue crescendo, a despeito de todos os esforços com seu tratamento ${ }^{3}$.

O objetivo do presente estudo consiste em verificar a relação entre obesidade e sintomas de depressão, ansiedade e desesperança em mulheres sedentárias e não sedentárias. 
Através deste estudo busca-se verificar as seguintes hipóteses:

1) A existência da relação entre obesidade e sintomas depressivos, ansiedade e desesperança;

2) Que esta relação é maior no grupo de mulheres sedentárias;

3) Quanto maior o nível de obesidade, maior a presença de sintomas depressivos, ansiedade e desesperança em ambos os grupos.

\section{2- MATERIAL E MÉTODOS}

\section{1- Sujeitos}

Participaram deste estudo de campo 40 mulheres, com idade entre 19 e 49 anos e idade média igual a 30,35 anos $( \pm 8,60)$. A coleta de dados foi realizada em uma academia de ginástica situada num bairro de classe média baixa da cidade de Ribeirão Preto - SP, mediante a devida autorização dos sujeitos e aprovação do Comitê de Ética em Pesquisa de uma instituição de ensino superior, conforme processo CEP - $\mathrm{n}^{\circ}$ 15/2006. Foram considerados para a análise de dados somente os sujeitos que não apresentaram nenhum problema de saúde grave, à exceção das patologias e distúrbios emocionais freqüentemente associados à obesidade, conforme relato verbal dos mesmos. Os sujeitos recrutados na academia foram divididos em dois grupos, de acordo com a prática ou não de exercício físico: grupo de mulheres não sedentárias (NS), caracterizadas pela prática de exercícios físicos há pelo menos três semanas consecutivas e no mínimo três vezes por semana e grupo de mulheres sedentárias ( $\mathrm{S}$ ), ou seja, que não praticavam qualquer tipo de exercício físico regular quando recrutadas após sua matrícula e avaliadas antes de iniciarem suas atividades na academia.

\section{2- Materiais e Instrumentos}

Os materiais e instrumentos utilizados para classificar os sujeitos consistiram em avaliações objetivas do humor, através do Inventário de Depressão Beck: Beck Depression Inventory (BDI), Inventário de Ansiedade Beck: Beck Anxiety Inventory (BAI) e Escala de Desesperança Beck: Beck Hopelessness Scale (BHS), seguidas de Avaliação Física, composta por duas partes: A) Identificação e B) Antropometria, descritos abaixo, de acordo com as variáveis consideradas para a análise dos dados:

\section{Sedentarismo - Avaliação Física/Parte A}

Identificação da idade da participante e do seu nível de condicionamento físico, através de um questionário fechado sobre a prática de atividade física, a fim de classificar a participante em sedentária ou não sedentária.

\section{Risco à saúde - Avaliação Física/Parte B}

Avaliação das circunferências da cintura e do quadril (em cm), com a utilização de uma fita métrica flexível, porém não elástica, incluída no item Perimetria, na Parte B da Avaliação Física. Estes dados foram utilizados para o cálculo da razão cintura quadril (RCQ) e sua classificação quanto ao risco à saúde 19. A RCQ consiste na circunferência da cintura dividida pela circunferência do quadril. A circunferência da cintura é definida como a circunferência mínima entre as costelas e a pelve e a medição do quadril é a circunferência máxima abaixo da cintura pélvica 20.

\section{Obesidade - Avaliação Física/Parte B}

Foram medidas a massa corporal total (em Kg) e a estatura (em m) de cada participante, através de uma balança de plataforma da marca Filizola ${ }^{\circledR}$, guarnecida por uma haste rígida e graduada para mensurar a estatura, estando o instrumento devidamente calibrado. Estes dados foram utilizados para o cálculo do IMC $\left(\mathrm{Kg} / \mathrm{m}^{2}\right)$ e sua classificação quanto à obesidade, de acordo com a Organização Mundial de Saú$\mathrm{de}^{21}$. Este índice pode ser obtido dividindo-se o peso corporal (em Kg) pelo quadrado da altura (em m), apresentando boa correlação com a adiposidade corporal ${ }^{5}$.

Também foram medidas as dobras cutâneas (em $\mathrm{mm}$ ) das participantes, utilizando o protocolo de três dobras para mulheres de Pollock et al ${ }^{22}$, a saber: tríceps, supra-ilíaca e coxa, para o cálculo da porcentagem de gordura corporal $(\% \mathrm{G})$ e sua classificação quanto à obesidade. $\mathrm{O}$ instrumento utilizado para medir as dobras cutâneas foi um plicômetro (compasso) clínico da marca CESCORF, com peso igual a $185 \mathrm{~g}$, com dimensões iguais a $286 \times 185 \mathrm{~mm}$, que possui um sistema simplificado de leitura, sensibilidade de $1 \mathrm{~mm}$ e boa precisão.

Os inventários BDI, BAI e BHS utilizados para as avaliações objetivas do humor, foram desenvolvidos por Beck e seus colaboradores no Center for Cognitive Therapy para avaliar aspectos da depressão, ansiedade e desesperança, respectivamente, traduzidos e validados para a população brasileira por Cunha $^{23}$. 


\section{Depressão - BDI}

O inventário de depressão Beck é composto por 21 itens, que se referem a: 1) tristeza; 2) pessimismo; 3) sentimento de fracasso; 4) insatisfação; 5) culpa; 6) punição; 7) auto-aversão; 8) auto-acusações; 9) idéias suicidas; 10) choro; 11) irritabilidade; 12) retraimento social; 13) indecisão; 14) mudança na autoimagem; 15) dificuldade de trabalhar; 16) insônia; 17) fatigabilidade; 18) perda de apetite; 19) perda de peso; 20) preocupações somáticas; 21) perda da libido. Cada qual com quatro alternativas de respostas, que podem ter escore $0,1,2$ ou 3. Obtém-se o escore total do BDI somando os escores de cada item correspondentes às alternativas assinaladas. A classificação da intensidade da depressão varia entre: 0 a 11 - mínimo; 12 a 19 - leve; 20 a 35 - moderado; 36 a 63 - grave ${ }^{23}$.

\section{Ansiedade - BAI}

O inventário de ansiedade Beck é uma escala de auto-relato composto por 21 itens, que representam quatro subescalas de sintomas: a) subescala fisiológica; b) subescala subjetiva; c) subescala de pânico e d) subescala autonômica. Cada item apresenta uma série escalar de 0 a 3 pontos que refletem níveis de gravidade crescente conforme os sintomas: 0 Absolutamente não; 1 - Levemente: não me incomodou muito; 2 - Moderadamente: foi muito desagradável, mas pude suportar; 3 - Gravemente: dificilmente pude suportar ${ }^{24}$. O escore total é o resultado da soma dos escores dos itens individuais, que representam os seguintes níveis de intensidade da ansiedade: 0 a 10 - mínimo; 11 a 19 - leve; 20 a 30 moderado; 31 a 63 - grave ${ }^{23}$.

\section{Desesperança - BHS}

A escala de desesperança Beck é uma medida da dimensão do pessimismo ou da extensão das atitudes negativas diante do futuro 25,26 , composta por 20 frases, nas quais o sujeito deve assinalar verdadeiro ou falso para cada afirmação apresentada. O escore total é o resultado da soma dos itens individuais, podendo variar de 0 a 20 e pontos de corte foram estabelecidos, indicando níveis de intensidade de desesperança: 0 a 4 - mínimo; 5 a 8 - leve; 9 a 13 - moderado e 14 a 20 - grave 23 .

\section{Procedimentos}

As avaliações foram realizadas em uma sala isolada, com boa iluminação e equipada com os materiais e instrumentos necessários, além de uma mesa e duas cadeiras. Inicialmente, os sujeitos foram submetidos individualmente às avaliações objetivas do humor, na seguinte ordem: BDI, BAI e BHS. As instruções consistiram em ler atentamente cada um dos itens e escolher a alternativa (BDI), assinalar a intensidade (BAI) ou circundar a letra C - certa ou E - errada (BHS) que correspondesse melhor aos pensamentos, emoções ou comportamentos que os sujeitos experimentaram nas últimas duas semanas.

Em seguida, os sujeitos foram submetidos à avaliação física, através da qual foram coletadas suas medidas de massa corporal total, estatura, circunferências da cintura e do quadril e espessura das dobras cutâneas: tríceps, supra-ilíaca e coxa. Os sujeitos foram pesados descalços, posicionados sobre a base da balança, com trajes leves. Antes da aferição do peso de cada participante, a balança foi previamente calibrada e sua precisão verificada por meio da pesagem de peso padronizado. A estatura foi mensurada por meio de haste graduada fixada em superfície plana, tendo em sua extremidade um marcador adaptável ao alto da cabeça. As participantes ficaram descalças, de costas para a haste, com os pés unidos, em posição ereta, com o olhar para o horizonte.

Para a tomada das medidas de circunferências, os sujeitos permaneceram na posição ereta, com os pés unidos, livres de suor ou pele coberta por óleos ou cremes. A fita métrica foi posicionada ao redor da cintura e posteriormente do quadril, estando o zero da fita na mão esquerda, exatamente acima ou abaixo da fita restante segurada pela mão direita do examinador, de modo a não pressionar essas regiões. A medida da circunferência foi aproximada para o meio centímetro mais próximo.

Quanto às dobras cutâneas, o examinador segurou o compasso com a mão direita, acionando o gatilho da garra móvel com o dedo indicador. Com os dedos polegar e indicador da mão esquerda, pinçouse a dobra cutânea, com a palma da mão voltada para o corpo do avaliado. O ponto tríceps foi medido por meio do pinçamento longitudinal, posicionando o compasso na região central entre o processo coracóide e o olécrano, estando o braço estendido. $\mathrm{O}$ ponto suprailíaca foi medido através do pinçamento oblíquo, posicionando o compasso sobre a linha média axilar, entre a última costela e a crista ilíaca. O ponto coxa foi medido através do pinçamento longitudinal, posicionando o compasso entre a espinha ilíaca anterior e a borda superior da patela, estando o sujeito sentado e com o joelho fletido em $90 \mathrm{o}$. Todas as medidas foram 
tomadas do lado direito do avaliado ereto, com um afastamento de cerca de $8 \mathrm{~cm}$ entre as pontas dos dedos indicador e polegar do pesquisador, estando as ponteiras cerca de $1,5 \mathrm{~cm}$ dos dedos que estavam realizando o pinçamento. Após o pinçamento a dobra foi solta e a leitura realizada. Estas medidas foram tomadas três vezes não consecutivas para cada dobra e considerada a média aritmética para posterior análise. O tempo total da coleta de dados foi aproximadamente 35 minutos.

Para a análise dos dados utilizou-se o Teste de Independência do $\mathrm{c}^{2}$, calculado pelo software Statistics e análises de regressão linear para mínimos quadrados, através da ferramenta de Análise de Dados do Excel.

\section{3- RESULTADOS E DISCUSSÃO}

A partir da classificação dos sujeitos em dois grupos, NS e S, tem-se a primeira análise dos dados para apresentação do perfil descritivo dos sujeitos quanto aos riscos à saúde (representados pela RCQ), grau de obesidade (representado pelo IMC e \%G) e intensidade das variáveis psicológicas: depressão, ansiedade e desesperança, indicada pelo escore total obtido no BDI, BAI e BHS para cada variável, respectivamente.

Quanto à variável RCQ, 50\% dos sujeitos do grupo NS apresentaram uma classificação alta para os riscos à saúde e somente 5\% apresentaram uma classificação do risco muito alto. Entretanto, no grupo $\mathrm{S}$, apesar de somente $45 \%$ dos sujeitos serem classificados com um risco alto à saúde, 20\% deles apresentaram um risco muito alto à saúde, corroborando os estudos de que pessoas sedentárias apresentam riscos mais elevados de desenvolver doenças crônicas quando comparadas às pessoas não sedentárias ${ }^{2,3,4}$.

Em relação à classificação da obesidade em função do IMC, os sujeitos com peso aceitável e com obesidade leve totalizaram $85 \%$ no grupo NS, contra $50 \%$ dos sujeitos do grupo $\mathrm{S}$, sendo que os outros $50 \%$ dos sujeitos apresentaram obesidade moderada e severa, contra $15 \%$ dos sujeitos do grupo NS. Quanto à variável \%G, 30\% dos sujeitos do grupo NS foram classificados entre os níveis de obesidade elevada e mórbida, contra $60 \%$ dos sujeitos do grupo $\mathrm{S}$ para os mesmos níveis de obesidade, sendo que $45 \%$ destes apresentaram obesidade mórbida. Estes resultados apóiam os estudos que consideram o sedentarismo como uma das principais causas da obesidade e que índices menores de IMC são encontrados em praticantes de atividade física regular ${ }^{2,4,5}$.

Quantos às variáveis psicológicas, pode-se observar que $75 \%$ dos sujeitos apresentaram intensidade mínima de depressão em ambos os grupos, porém $20 \%$ dos sujeitos do grupo $\mathrm{S}$ apresentaram intensidade moderada de depressão contra 5\% dos sujeitos do grupo NS. Em relação à ansiedade, somente $15 \%$ dos sujeitos do grupo NS apresentaram intensidades leve ou moderada, enquanto $25 \%$ dos sujeitos do grupo S apresentaram intensidades leve, moderada ou grave. Estes resultados corroboraram achados quanto à presença de maiores níveis de depressão e ansiedade em pacientes obesos ${ }^{9,10}$, devido à maior incidência de mulheres obesas no grupo S, no presente estudo. Os dados encontrados apoiaram também a possibilidade da existência de uma relação diretamente proporcional entre a presença destes sintomas e o IMC $^{9}$. Ou seja, quanto maior o IMC, maior a presença de sintomas depressivos e ansiógenos.

Em contrapartida, sobre a presença de desesperança, nota-se uma divergência em relação aos grupos, já que 35\% dos sujeitos do grupo NS apresentaram intensidade leve e 5\% grave, enquanto $20 \%$ dos sujeitos do grupo $\mathrm{S}$ apresentaram intensidade leve e $15 \%$ moderada, tornando delicada qualquer tipo de análise inicial quanto a esta variável.

Com o propósito de verificar se há uma relação de dependência entre as variáveis deste estudo, os dados foram submetidos ao Teste de Independência de Qui-Quadrado $(\div 2)$, calculado pelo software Statistics, para os dois grupos (NS e S), cruzando as variáveis RCQ, IMC, \%G, BDI, BAI e BHS.

$\mathrm{O}$ teste do $\div \mathbf{2}$, para $\mathbf{p}<0,05$, produziu uma diferença significativa entre todos os cruzamentos para ambos os grupos, com exceção do cruzamento $\% \mathrm{G} \mathrm{x}$ BAI: $\div 2(20)=27,07 ; \mathbf{p}=0,133$ para o grupo $S$.

Esses resultados implicam rejeição da hipótese de independência entre as variáveis, ou seja, as classificações da RCQ, do IMC, da \%G e das variáveis psicológicas influenciam umas às outras, apresentando, portanto, uma relação de dependência, tanto em mulheres sedentárias como em mulheres não sedentárias. Esses dados apóiam os estudos mostrando a relação direta entre o grau de obesidade e risco à saúde 2,3,4,5,6 e a relação entre obesidade e presença de sintomas de depressão e ansiedade ${ }^{8,9,10,13,14,15}$.

Em seguida, foram realizadas análises de regressão linear para mínimos quadrados, através da ferramenta de Análise de Dados do Excel, a fim de 
Tabela I: Resultados do teste do $\div 2$ para os cruzamentos entre as variáveis RCQ, IMC, \% G, BDI, BAI e BHS para os grupos NS e S.

\begin{tabular}{llllll}
\hline \multirow{2}{*}{ Cruzamentos } & \multicolumn{2}{c}{ NS } & & \multicolumn{2}{c}{$\mathrm{S}$} \\
\cline { 2 - 3 } \cline { 5 - 6 } & $\div 2$ & $\mathrm{p}$ - Significância & & $\div 2$ & $\mathrm{p}$ - Significância \\
\hline RCQ/BDI & 22,73 & $0,007^{*}$ & & 25,52 & $0,013^{*}$ \\
RCQ/BAI & 25,53 & $0,002^{*}$ & & 28,12 & $0,031^{*}$ \\
RCQ/BHS & 24,29 & $0,004^{* *}$ & & 27,74 & $0,006^{*}$ \\
IMC/BDI & 23,12 & $0,006^{*}$ & & 28,05 & $0,005^{*}$ \\
IMC/BAI & 25,53 & $0,002^{*}$ & & 44,92 & $0^{*}$ \\
IMC/BHS & 23,5 & $0,005^{*}$ & & 32,12 & $0,001^{*}$ \\
\%G/BDI & 34,26 & $0,0038^{*}$ & & 29,56 & $0,014^{*}$ \\
\%G/BAI & 29,112 & $0,016^{*}$ & & 27,07 & 0,133 \\
\%G/BHS & 26,83 & $0,03^{*}$ & & 32,73 & $0,005^{*}$ \\
BDI/BAI & 33,45 & $0^{*}$ & & 38,36 & $0^{*}$ \\
BDI/BHS & 31,07 & $0^{*}$ & & 37,34 & $0^{*}$ \\
BAI/BHS & 32,07 & $0^{*}$ & & 38,91 & $0^{*}$ \\
\hline
\end{tabular}

* Significativo a $5 \%$.

confirmar a presença de dependência entre as variáveis estudadas para cada grupo, na forma: - BDI como variável dependente e como variáveis independentes: RCQ, IMC e \% G; - BAI como variável dependente e como variáveis independentes: RCQ, IMC e \% G;
- BHS como variável dependente e como variáveis independentes: RCQ, IMC e \% G.

Os resultados podem ser visualizados nas tabelas a seguir, para cada grupo, NS e S, respectivamente.

Tabela II: Resultados do ajuste do modelo de regressão linear por mínimos quadrados, considerando as variáveis respostas BDI, BAI e BHS para o grupo NS.

\begin{tabular}{ccccc}
\hline Variável resposta & \multicolumn{2}{c}{ Variável independente } & P- Significância & $\mathrm{R}^{2}$ - Coeficiente explicativo \\
\hline BDI & RCQ & 13,176 & 0,750 & 0,006 \\
& IMC & 0,596 & 0,141 & 0,116 \\
& $\% G$ & 0,385 & 0,064 & 0,177 \\
\multirow{2}{*}{ BAI } & RCQ & $-16,216$ & 0,729 & 0,007 \\
& IMC & 0,408 & 0,384 & 0,042 \\
& $\%$ G & 0,282 & 0,246 & 0,074 \\
BHS & RCQ & $-13,851$ & 0,491 & 0,027 \\
& IMC & 0,004 & 0,986 & 0,000 \\
& $\%$ GG & 0,104 & 0,322 & 0,054 \\
\hline
\end{tabular}


Os resultados da análise de regressão linear por mínimos quadrados, apresentados nas Tabela II, desconfirmaram a relação de dependência entre as variáveis estudadas, conforme mostrada no teste do $\div 2$ para o grupo NS. Ou seja, não existe relação de dependência entre as variáveis em questão neste grupo. Isto implica que a presença e a intensidade das variáveis psicológicas não dependem do grau de obesidade e dos riscos à saúde e vice-versa, em mulheres não sedentárias, isto é, que praticam algum tipo de atividade física regular.

Estes dados podem indicar que a prática de atividade física neste grupo é um fator de minimização de sintomas psíquicos, contribuindo, assim, para o bemestar físico e mental destes sujeitos. Tal observação vem corroborar a idéia de que a prática de atividade física favorece não só a saúde física ${ }^{2,3}$, como a saúde mental das pessoas em geral ${ }^{3,11,12}$.

Já os resultados desta mesma análise para o grupo S confirmaram a existência da relação de dependência entre as variáveis estudadas, conforme os valores indicados na Tabela III, desconfirmando a não dependência para o cruzamento $\% \mathrm{G}$ x BAI revelada no teste do $\div 2$. Exceções ocorreram para os cruzamentos: BAI x RCQ e BHS x \% G desconfirmando, assim, a relação de dependência para estes pares, conforme o teste do $\div 2$.

Estes resultados, à exceção dos cruzamentos BAI x RCQ e BHS x \% G, indicam que, apesar do coeficiente de explicação $(\mathbf{R} 2<85 \%)$ não ser signifi- cativo, a análise de regressão linear permite afirmar que as variáveis psicológicas dependem do grau de obesidade e risco à saúde e vice-versa, presentes em mulheres sedentárias, ao contrário do grupo NS.

É importante observar que o fato de uma pequena, porém significativa, porcentagem da variabilidade de depressão, ansiedade e desesperança ser explicada pelas variáveis RCQ, IMC e \%G implica existência de outros fatores que interferem neste modelo, que não foram abordados. Tal limitação do presente estudo se justifica pela própria multiplicidade e complexidade dos fatores associados à obesidade ${ }^{1,2,4,5,9}$.

Estes dados vêm corroborar os estudos, citados anteriormente, que encontraram a presença de níveis de depressão e ansiedade maiores em pacientes obesos e a necessidade de incluir, no tratamento da obesidade, não só a prática de atividade física regular, como também a psicoterapia, em especial a terapia cognitiva, que se mostrou eficiente nesses casos, conforme vários estudos controlados e observações clínicas ${ }^{8,13-17}$. Portanto, acredita-se que esse tratamento conjunto, somado à dieta alimentar adequada e orientada, possa ser uma das ferramentas preditoras de sucesso no tratamento e prevenção à obesidade.

Observa-se, ainda, através da análise de regressão, que a relação de dependência entre as variáveis é crescente, ou seja, à medida que aumentam os níveis de depressão, ansiedade e desesperança, aumentam também os graus dos riscos à saúde e da obesidade

Tabela III: Resultados do ajuste do modelo de regressão linear por mínimos quadrados, considerando as variáveis respostas BDI, BAI e BHS para o grupo $\mathrm{S}$.

\begin{tabular}{|c|c|c|c|c|}
\hline \multirow{2}{*}{$\begin{array}{r}\text { Variável } \\
\text { BDI }\end{array}$} & \multicolumn{2}{|c|}{ Variável independente } & \multirow{2}{*}{$\begin{array}{c}\text { P-Significância } \\
0,035^{*}\end{array}$} & \multirow{2}{*}{$\frac{\text { R2-Coeficiente explicativo }}{0,223}$} \\
\hline & RCQ & 75,123 & & \\
\hline & IMC & 0,927 & $0,009 *$ & 0,325 \\
\hline & $\% \mathrm{G}$ & 0,497 & $0,019 *$ & 0,267 \\
\hline \multirow[t]{3}{*}{ BAI } & RCQ & 45,014 & 0,275 & 0,066 \\
\hline & IMC & 1,015 & $0,009 *$ & 0,320 \\
\hline & $\% \mathrm{G}$ & 0,498 & $0,037^{*}$ & 0,221 \\
\hline \multirow[t]{3}{*}{ BHS } & RCQ & 33,901 & $0,025^{*}$ & 0,250 \\
\hline & IMC & 0,319 & $0,041 *$ & 0,212 \\
\hline & $\% G$ & 0,128 & 0,179 & 0,098 \\
\hline
\end{tabular}

*Significativo a $5 \%$. 
em ambos os grupos. Exceção para os cruzamentos BAI x RCQ e BHS x RCQ no grupo NS, onde ocorreu uma relação inversa. Tal relação de dependência foi significativa apenas para o grupo $\mathrm{S}$.

Os gráficos a seguir, representados pelas Figuras 1, 2, 3, 45 e 6, ilustram a relação de dependência entre as variáveis, através da apresentação dos escores atribuídos a cada variável psicológica em função dos valores encontrados para as variáveis RCQ, IMC e $\% \mathrm{G}$ para cada grupo estudado.

Apesar da não existência de relação de dependência entre as variáveis para o grupo NS, pode-se observar uma curva suavemente crescente para todas as combinações de variáveis, indicando o aumento da intensidade de todas as variáveis psicológicas em função do IMC e da \%G e em BDI x RCQ, o que não ocorreu nas combinações BAI x RCQ e BHS x RCQ, nas quais se observa uma curva ligeiramente decrescente, conforme representadas nas Figuras 1, 2 e 3.

Ao comparar os dados das Figuras 1, 2 e 3 com os dados das Figuras 4, 5 e 6, é possível observar claramente a presença de curvas mais acentuadas para todas as combinações de variáveis, de forma a ilustrar a relação crescente e significativa de dependên-

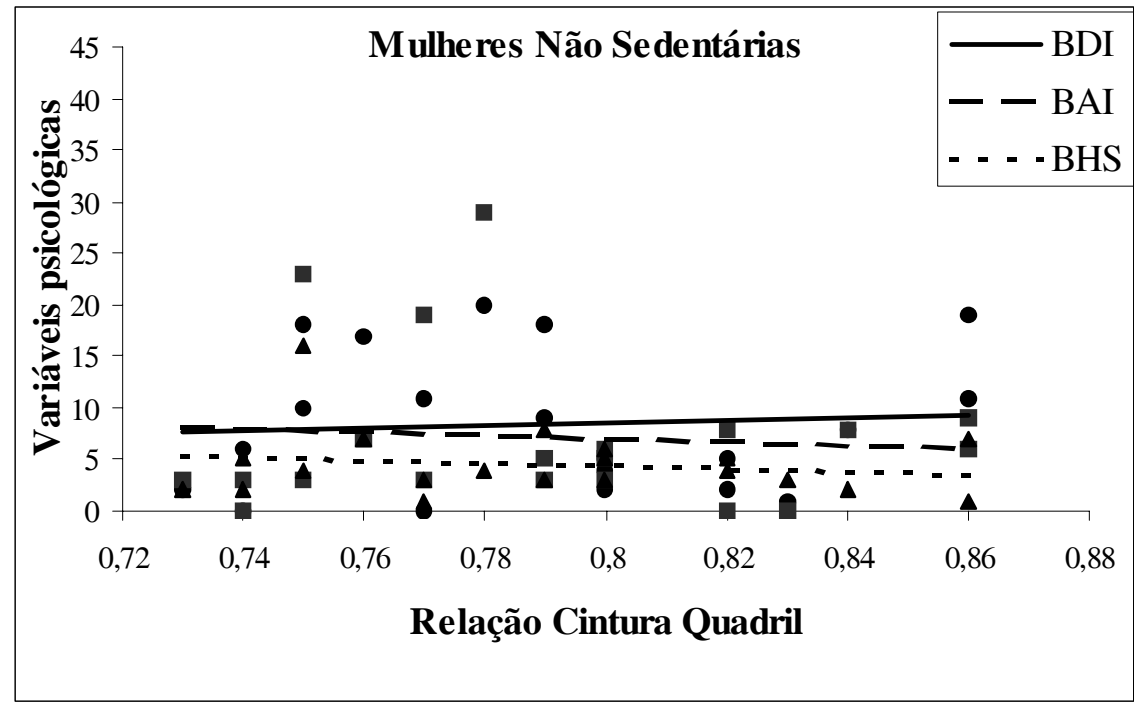

Figura 1: Níveis de depressão (BDI), ansiedade (BAI) e desesperança (BHS) em função da RCQ para o grupo NS.

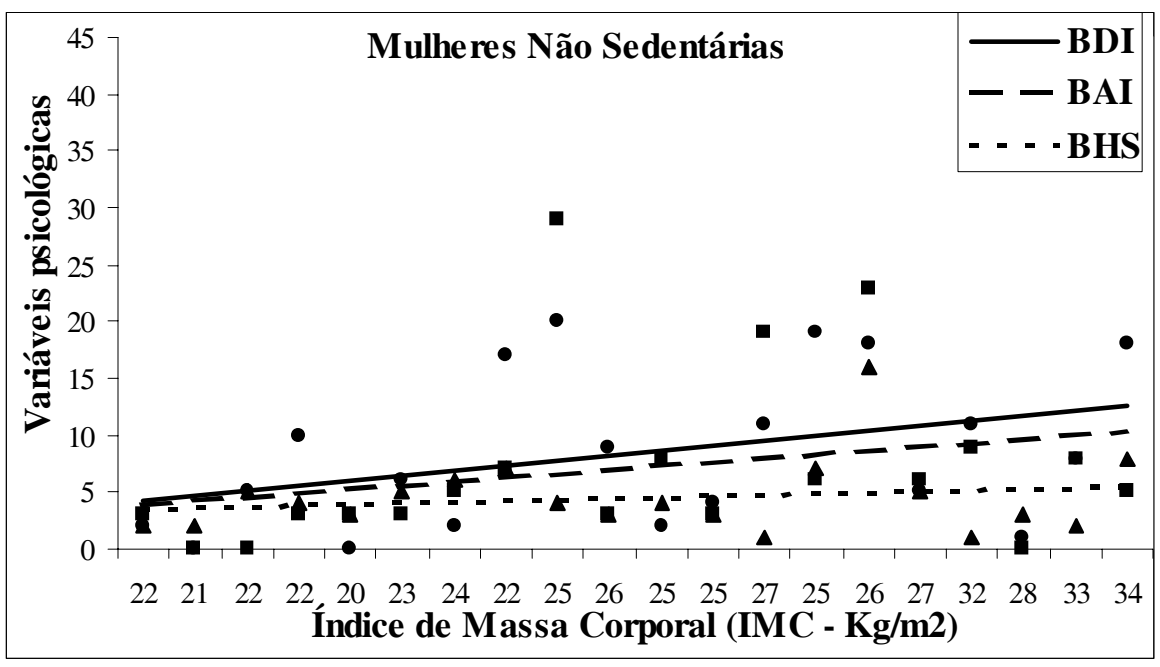

Figura 2: Níveis de depressão (BDI), ansiedade (BAI) e desesperança (BHS) em função do IMC $(\mathrm{Kg} / \mathrm{m} 2)$ para o grupo NS. 
cia das variáveis no grupo $\mathrm{S}$, com exceção dos pares BAI x RCQ e BHS x $\% \mathrm{G}$, na qual não foi confirmada tal relação, apesar da tendência crescente de suas curvas.

\section{5- CONCLUSÕES}

Os dados apresentados neste estudo sustentaram as hipóteses iniciais quanto à existência da relação entre obesidade e sintomas depressivos, ansiógenos e de desesperança somente no grupo de mulheres sedentárias, já que esta relação de dependência não pôde ser confirmada no grupo de mulheres não sedentárias. Os dados demonstram também que, quanto maior o nível de obesidade, maiores os níveis dos sintomas psíquicos, tanto para as mulheres sedentárias quanto para as mulheres não sedentárias.

Apesar de a variável riscos à saúde não ter sido abordada nas hipóteses iniciais deste estudo, análises comprovaram sua relação direta com a obesidade e a relação de dependência com as variáveis depressão e desesperança, no grupo de mulheres sedentárias.

Essas observações parecem indicar que a prática de atividade física foi um fator de minimização da presença e da intensidade de depressão, ansiedade e desesperança em mulheres não sedentárias. Porém, uma discussão qualitativa desses dados não permite afirmar que essas relações são de causa e efeito de certas variáveis em relação às outras, justificando a necessidade de investigar como essas e outras variáveis se manifestam nos diferentes graus de obesidade e níveis de condicionamento físico.

Enfim, entende-se que a prática de exercícios físicos, juntamente com o acompanhamento nutricional e a psicoterapia, podem ser considerados importantes instrumentos no combate à obesidade e patologias associadas.

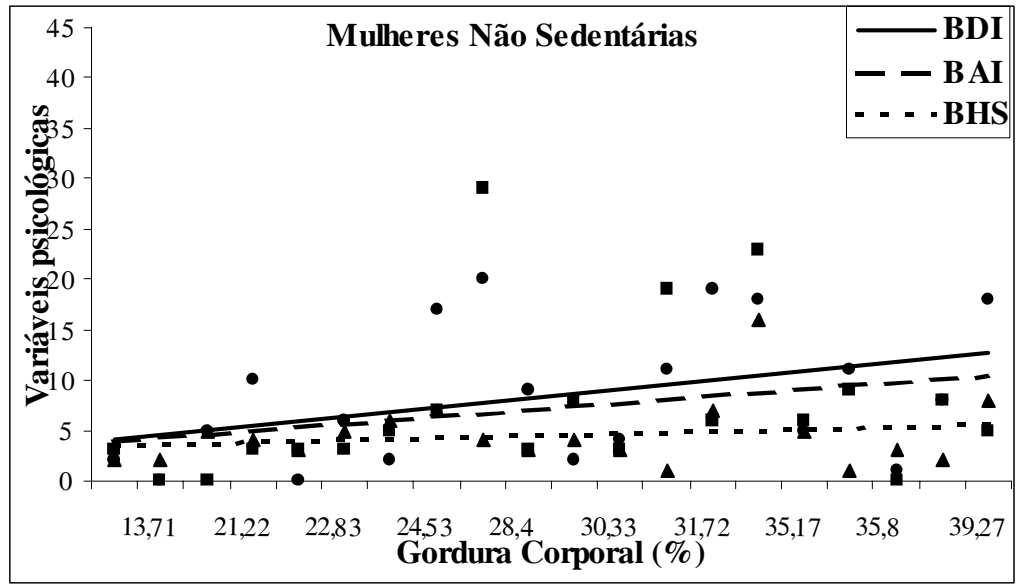

Figura 3: Níveis de depressão (BDI), ansiedade (BAI) e desesperança (BHS) em função da \%G para o grupo NS.

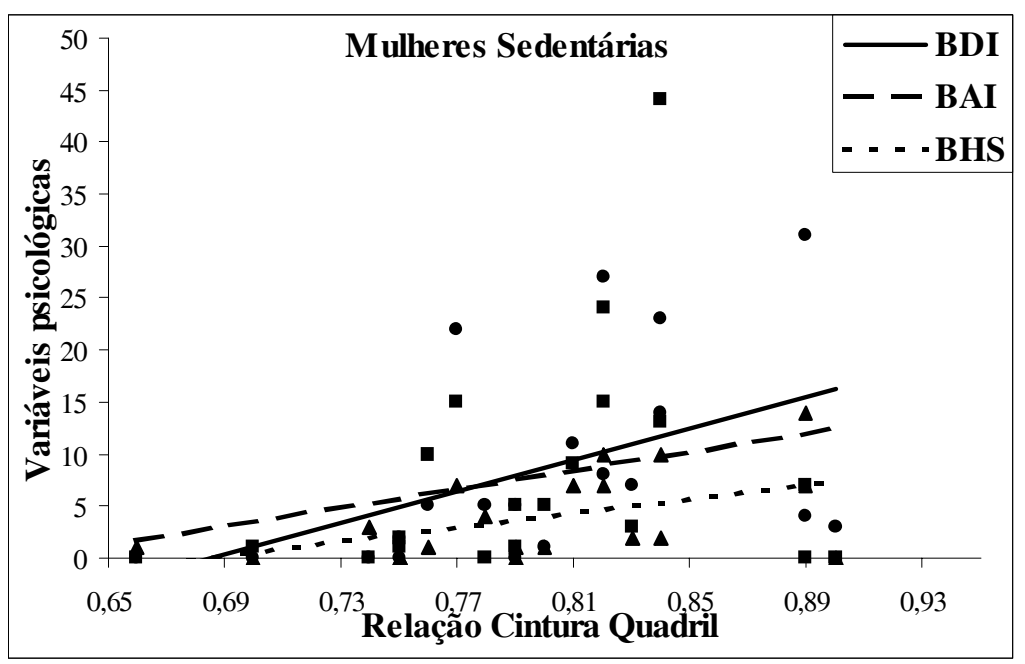

Figura 4 - Níveis de depressão (BDI), ansiedade (BAI) e desesperança (BHS) em função da RCQ para o grupo S.

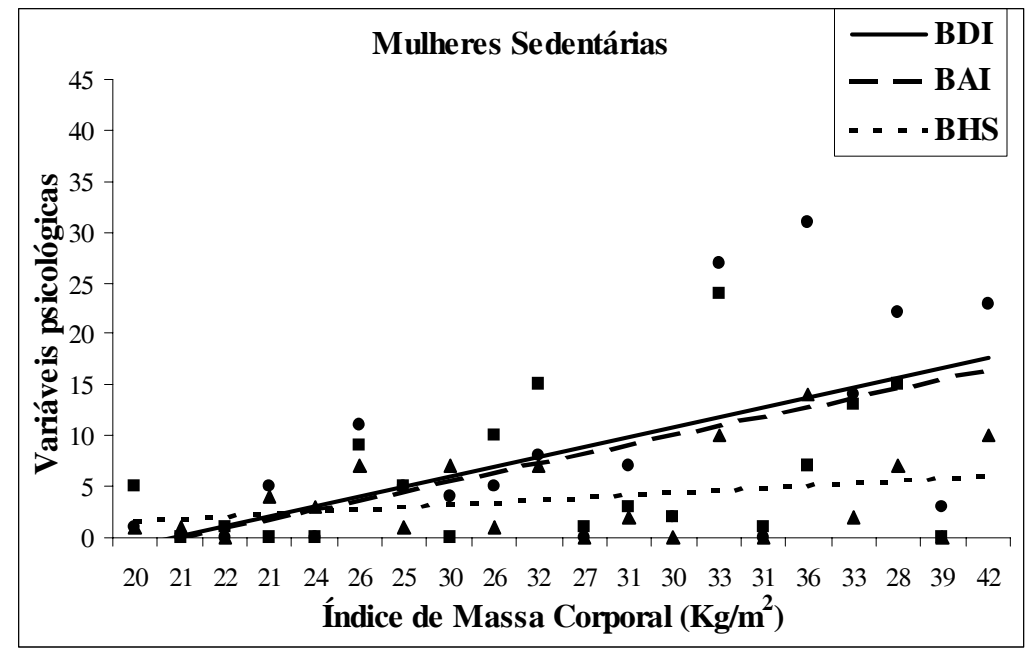

Figura 5 - Níveis de depressão (BDI), ansiedade (BAI) e desesperança (BHS) em função do IMC (Kg/m2) para o grupo $S$. 


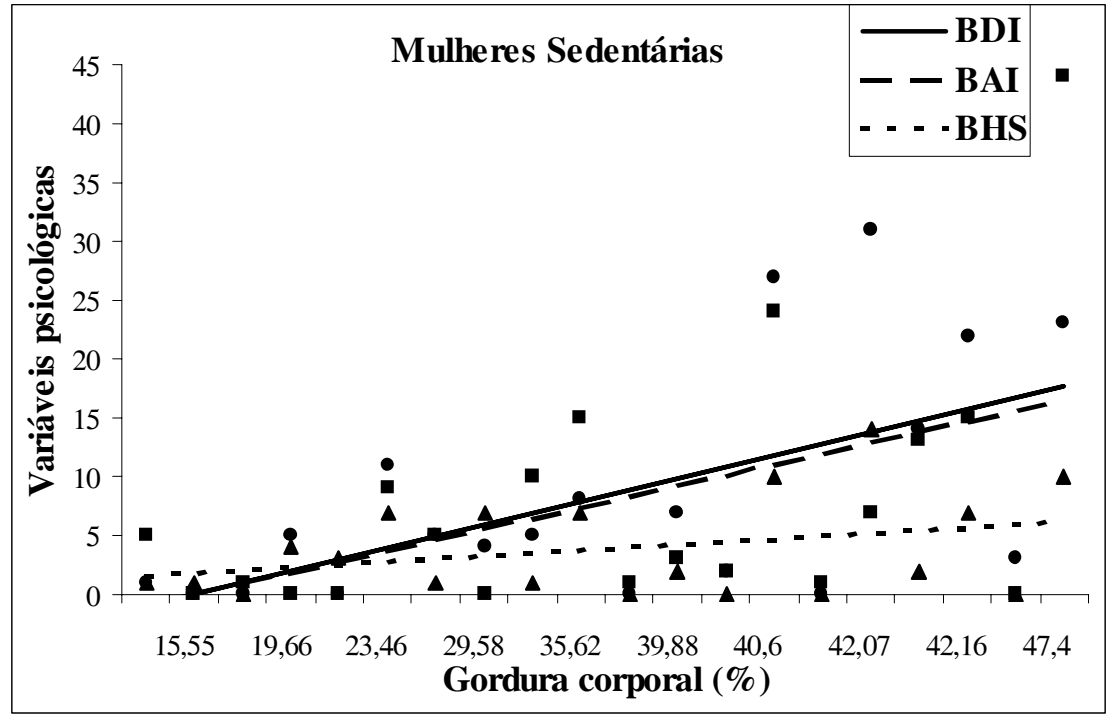

Figura 6: Níveis de depressão (BDI), ansiedade (BAl) e desesperança (BHS) em função da $\% \mathrm{G}$ para o grupo $\mathrm{S}$.

Tosetto AP, Simeão Júnior CA. Obesity and symptoms of depression, anxiety and hopelessness in sedentary and non-sedentary women. Medicina (Ribeirão Preto) 2008; ; 41 (4): 497-507.

ABSTRACT: The aim of this field study was to verify if there is a relation between obesity and symptoms of depression, anxiety and hopelessness in 40 women aged 30,35 on average ( \pm $8,60)$, divided into two groups: non-sedentary ones, characterized for doing a physical activity at least three times a week for three weeks in a row and sedentary ones, characterized by not practicing any type of regular physical activity when recruited. The method consisted of: objective evaluations of humor, through Beck Inventories of Anxiety (BAI), Depression (BDI) and Hopelessness (BHS) and Physical Evaluation, including total body mass, height, waist and hip circumferences and skin folds thickness. Calculations of the body mass index (BMI), of the waist/ hip index (WHI) and of the percentage of corporal fat (\%F) were performed in order to evaluate the presence and level of obesity. Results of the analysis of regression to square minimum supported the initial hypothesis concerning the existence of a relation between obesity and psychic symptoms only in sedentary women (BDI/WHI, $p=0,035$, BDI/BMI, $p=0,009, B D I / \% G, p=0,019, B A I / B M I, p=0,009$, $\mathrm{BAI} / \% \mathrm{G}, \mathrm{p}=0,037, \mathrm{BHS} / \mathrm{WHI}, \mathrm{p}=0,025, \mathrm{BHS} / \mathrm{BMI}, \mathrm{p}=0,041)$, once the relation of dependency could not be confirmed in non-sedentary women BDI/WHI, $p=0,750$, BDI/BMI, $p=0,141$, BDI/\%G, $p=0,064$, BAI/WHI, $\mathbf{p}=0,729$, BAl/BMI, $\mathbf{p}=0,384, \mathrm{BAI} / \% \mathrm{G}, \mathbf{p}=0,246, \mathrm{BHS} / \mathrm{WHI}, \mathbf{p}=0,491, \mathrm{BHS} / \mathrm{BMI}, \mathbf{p}=0,986$, $\mathrm{BHS} / \% \mathrm{G}, \mathbf{p}=0,322)$ and the greater the level of obesity, the greater the level of psychic symptoms in both groups. These observations seem to point out that the practice of physical activities was a factor of minimization of presence and intensity of psychic symptoms in non-sedentary women.

Keywords: Obesity. Symptoms, Psychic. Physical Activity. 


\section{REFERÊNCIAS}

1 - Pinheiro ARO, Freitas SFT, Corso ACT. Uma abordagem epidemiológica da obesidade. Rev Nutr, Campinas, 2004; 17(4): 523-33.

2 - Damaso AR, Guerra RLF. A atividade física espontânea no tratamento da obesidade. Rev ABESO, 2002 abr; III(7). Avaliável on-line: http://www.abeso.org.br/revista/revista7/ educ_fis.htm. Acesso em 25 nov 2005.

3 - Coutinho WF (org.). Documento do Consenso Latino-Americano em Obesidade. Avaliável on-line: http:// www.emagrecentro.com.br/news/consenso2.pdf. Acesso em: 19 mar 2006.

4 - Instituto Nacional de Cardiologia. Avaliável on-line: http:// www.incl.rj.saude.gov.br/incl/paginas/fat.asp\#2. Acesso em: 23 mar 2007.

5 - Mancini M. Métodos de avaliação de obesidade e alguns dados epidemiológicos. Rev ABESO, III(11), dez 2002. Avaliável on-line: http://www.abeso.org.br/revista/revista11/ metodos.htm. Acesso em 28 nov. 2005.

6 - Monteiro CA. (org.). Velhos e novos males da saúde no Brasil: a evolução do país e de suas doenças. São Paulo: Hucitec e Nupens / USP; 1995.

7 - Halpern A, Monegaglia AP, Beyruti M. Obesidade. Rev ABESO, 20. Avaliável on-line: http://www.abeso.org.br/revista/revista20/obesidade.htm. Acesso em 25 nov. 2005.

8 - Beck JS. Terapia Cognitiva: Teoria e Prática. Porto Alegre: Artes Médicas; 1997.

9 - Segal A. Aspectos psiquiátricos da obesidade. Rev ABESO, IV(12), fev 2003. Avaliável on-line: http://www.abeso.org.br/ revista/revista12/aspectos.htm. Acesso em 25 nov. 2005.

10 - Matos MIR, Zanella MT. Alterações do comportamento alimentar, ansiedade, depressão e imagem corporal em pacientes como obesidade grau III. Rev ABESO, III(9), Ag 2002. Avaliável on-line: http://www.abeso.org.br/revista/revista9/ alteracoes.htm. Acesso em: 28 nov. 2005.

11 - Paffenbarger RS Jr, Hyde RT, Wing AL, Hsieh CC. Physical activity, all-cause mortality, and longevity of college alummi. N Engl J Med. 1986; 6; 314(10):605-13.

12 - Pate R, Pratt M, Blair SN, Haskell WL, Macera CA, Bouchard $C$ et al. Physical activity and public health. A recommendation from the Centers for Disease Control and Prevention and the American College of Sports Medicine. JAMA, 1995; 273: $402-7$.

13 - Keller, C, Oveland, D, Hudson, S. Strategies for Weight Control Success in Adults. Nurse-Pract, 1997; 22(3): 33, 37-8.

14 - Tanco, S, Linden, W, Earle, T. Well-Being and morbid obesity in women: a controlled therapy evaluation. Int-J-EatingDisord; 1998; 23(3):325-39.
15 - World Health Organization. Obesity: preventing and managing the global epidemic. Report of a WHO consultation. World Health Organ Tech Rep Ser; 2000; 894:i-xii, 1-253.

16 - Kirschenbaum, DS, Johnson, WG, Stalonas, PM. Treating Childhood and Adolescent Obesity. NY: Pergamon Press, 1987.

17 - Duchesne M, Appolinario JC, Range BP, Fãndino J, Moya T, Freitas SR. Utilização de terapia cognitivo-comportamental em grupo baseada em manual em uma amostra brasileira de indivíduos obesos com transtorno da compulsão alimentar periódica. Rev. Bras. Psiquiatr.; 2007; 29(1): 23-25.

18 - Duchesne M, Appolinario JC, Range BP, Freitas S, Papelbauml, $M$, Coutinho W. Evidências sobre a terapia cognitivo-comportamental no tratamento de obesos com transtorno da compulsão alimentar periódica. Rev. Psiquiatr. Rio Gd. Sul; 2007; 29 (1): 80-92.

19 - Heyward, VH, Stolarczyk, LM. Applied body composition assessment. Champaign, IL: Human Kinetics. 1996. Avaliável on-line: http://www.saudeemmovimento.com.br/saude/ avaliation_fisica_i.htm. Acesso em 11 out. 2006.

20 - Tritschler, KA. Medida e Avaliação em Educação Física e Esportes de Barrow \& McGee. 5.a edição. Tradução de Márcia Greguol. Barueri, SP: Manole, 2003.

21 - Organização Mundial de Saúde. Classificações para o índice de massa corpórea - IMC. em adultos 2002. Avaliável online: http://www.saudeemmovimento.com.br/saude/tabelas/ tabela_de_referencia_imc.htm. Acesso em: 11 out. 2006.

22 - Pollock, ML, Heyward, VH, Petroski, EL., Siri, WE. Avaliador da composição corporal - Protocolo de 3 dobras para MuIheres Adultas 2001. Avaliável on-line: http:// www. saudeem movimento.com.br/saude/ avaliation_fisica_i.htm Acesso em 25 nov. 2005.

23 - Cunha, JA. Manual da versão em português das Escalas Beck. São Paulo: Casa do Psicólogo, 2001.

24 - Beck, AT, Steer, RA. Beck Anxiety Inventory. Manual. San Antonio, TX: Psychic Corporation, 1993a.

25 - Beck, AT, Steer, RA. Beck Hoppelessness Scale. Manual. San Antonio, TX: Psychic Corporation, 1993b.

26 - Beck, AT. et al. The measurement of pessimism: the Hoppelessness Scale. J Consul Clin Psychol, 1974; 42(6): 861-865.

Recebido para publicação em 03/03/2008

Aprovado para publicação em 01/10/2008 